\title{
Foam Rolling as a Warm-up Technique for Anaerobic Power Activities
}

\author{
D'Andrea JD, Wicke J* and Kleber F
}

Kinesiology Department, William Paterson University, USA

*Corresponding author: Jason Wicke, Kinesiology Department, William Paterson University, 200 Pompon Road, Wayne, NJ, 07481, USA, Tel: 973-720-3271, Fax:973-720-2034,E-mail:wickej@wpunj.edu

\begin{abstract}
The purpose of this study was to examine the effects of Foam Rolling (FR) compared to an Active Warm-Up (AWU) on performance measures of anaerobic power. Twenty-one male participants, recruited from a Division III university's athletic teams, completed a two day cross-over protocol to examine knee extension output using an isokinetic dynamometer. Peak torque, average peak torque, total work, and average power measurements were taken based on one set of 5 maximal concentric contractions of the quadriceps pre and post-intervention of one of the warm-up procedures (FR vs. AWU). Following a pre-testing, subjects were randomly assigned to complete either the FR or AWU warm-up on the first day. The FR protocol involved 3 sets of 30 -second rolling on 3 zones of the thigh (medial, anterior, lateral); the AWU warm-up included 3 sets of 30 seconds each of high knees, anterior-posterior leg swings, and lateral leg swings. Immediately following the warm-up protocol, the isokinetic measurements were repeated. The following week, subjects repeated the pre and post-testing but with the other warm-up protocol. Statistical analyses using repeated measure t-tests indicate significant increases $(p<$ 0.01 ) in peak torque following both the foam rolling and the active warm-up protocols. There were no significant differences on the four performance measures between the changes (post minus pre-testing) for FR compared to AWU. These results suggest that foam rolling could be used to increase performance when used as a warm-up modality.
\end{abstract}

\section{Keywords}

Dynamic stretching, Torque, Work

\section{Introduction}

Over the decades, coaches, exercise professionals, and instructors alike have recommended a proper warm-up to prepare for physical activity. A traditional warm-up has many goals such as increased body temperature, in- creased range of motion, and ultimately increased performance. With more research on the various styles of warm-ups, it has been shown that partaking in a thorough warm-up can actually increase performance and decrease the risk of injury [1-4]. Stretching, as a component of a warm-up, has been thought to reduce risk of injury by allotting each joint a greater range of motion [3-5], though static stretching specifically is thought to reduce power output $[6,7]$. Because warm-ups are very sport specific and rely on mostly trial and error, it can be very difficult to find evidence that a certain warm-up is effective for all areas of physical activity $[2,3,8]$.

Manual therapy techniques, which include hands on techniques like myofascial release and massage, can be used for a variety of circumstances. Specifically, myofascial release has been historically used to alleviate pain within the muscular and fascia tissue and promote healing during rehabilitation [9]. More recently, myofascial release and Self-Myofascial Release (SMR) have become a more common pre-competition modality to increase performance. Foam rolling has been a common modality in these novel SMR techniques, but there is limited evidence to show that foam rolling is a true myofascial release therapy. Still, most areas of sport medicine are using the terms foam rolling and self-myofascial release interchangeably.

Fascia, the connective tissue that surrounds muscles and its tendons, works as a supporting barrier for the muscle fibers and helps attach tendons to bone. Fascia has a unique property that allows it to become more pliable the more it is used or disturbed; the more pliable the fascia is, the more movement it will allow [10].

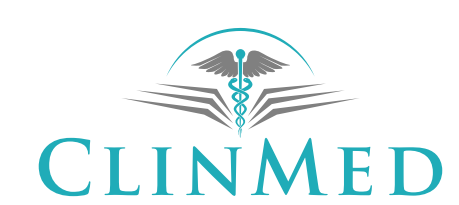

INTERNATIONAL LIBRARY 
However, there is a disadvantage to this thixotropic characteristic: If a muscle lies immobile for a period of time, the fascia surrounding that muscle can become non-compliant and rigid. This rigidity can cause a decreased range of motion and even an inflammatory response between the muscle and its fascia [11,12]. Myofascial release (MFR) therapies have been designed to mobilize the fascia to make it more compliant and allow a greater range of motion.

The process of Foam Rolling (FR) is truly unique and cannot be placed into a simple category of therapy. The pressure exerted by the foam roller can cause physiological responses similar to massage as well as assist with breaking up adhesions similar to MFR. FR has muscle lengthening effects following a one-minute bout of using a body weight high-density roller [13]. In addition, immediate results of increased range of motion has been shown with varying forms of stretching in conjunction with foam rolling, but the greatest increase typically involves some form of static stretching which may be the dominating factor [13]. Foam rolling, therefore, can be described as a combination of massage, MFR, and static stretching. So far, it has been noted that these three forms of therapy, when evaluated separately, show a trend toward decreased power and anaerobic performance $[6,7,12,14]$. However, there is limited research on FR separately and its effects on muscle strength and power output. Furthermore, the extent to which FR mimics each of the three therapies listed above is yet unknown.

Foam rolling is still a relatively novel concept and therefore does not have a standardized protocol for use. Research designs have adopted foam rolling protocols ranging from 10 seconds to 1 minute bouts $[9,13,15]$. Generally multiple sets were used, such that the total time of foam rolling, excluding rest times, ranged from 30 seconds to 6 minutes. The minimum amount of time spent foam rolling that has shown to provoke physiological changes is 30 continuous seconds.

With the limited research on foam rolling as a modality for performance, is it still undetermined on how a foam roller's physical design could affect the patient's deep tissue fascia [16]. Originally thought to reduce pain and stiffness resulting from muscular adhesions, foam rolling has since been shown to create an increased vascular response [17]. Due to the vasodilation response recorded after foam rolling, it has since been theorized that foam rolling could provide performance enhancing benefits and thus be utilized during a warm-up $[9,17]$. No significant detriments on performance have been shown with the implementation of FR $[13,15]$.

It appears there is potential for the use of foam rolling as a warm-up modality. Although the concept of foam rolling is similar to stretching, myofascial release, and massage, they do not elicit similar results on performance. The mechanisms behind foam rolling are still undetermined, but with the possible utilization of the autogenic and reciprocal reflexes, foam rolling's effect on the body could be similar to a dynamic stretch. The purpose of this study is to explore the potential of foam rolling as a warm-up technique.

\section{Methods}

\section{Experimental approach to the problem}

Differences between the pre-test and post-test measures on the dependent variables of average torque, peak torque, total work and average power were recorded. The two independent variables included a foam rolling protocol versus a traditional active warm-up protocol. The testing days were one week apart with each session occurring at approximately the same time of day.

\section{Subjects}

Male student athletes $(N=21)$ were recruited from soccer, basketball and football varsity teams within a Division III, four-year institution in New Jersey. Subjects were (average \pm standard deviation) $21.1 \pm 2$ years of age, weighed $90.5 \pm 12.5 \mathrm{~kg}$, and $179.6 \pm 7.6 \mathrm{~cm}$ tall. Prior to volunteering, subjects signed an informed consent form approved by the Institutional Review Board of the institution, and completed the Physical Activity Readiness Questionnaire (Par-Q). The Par- $Q$ was used to determine if the subjects had a medical or physical conditions that would contraindicate physical exertion (Figure 1).

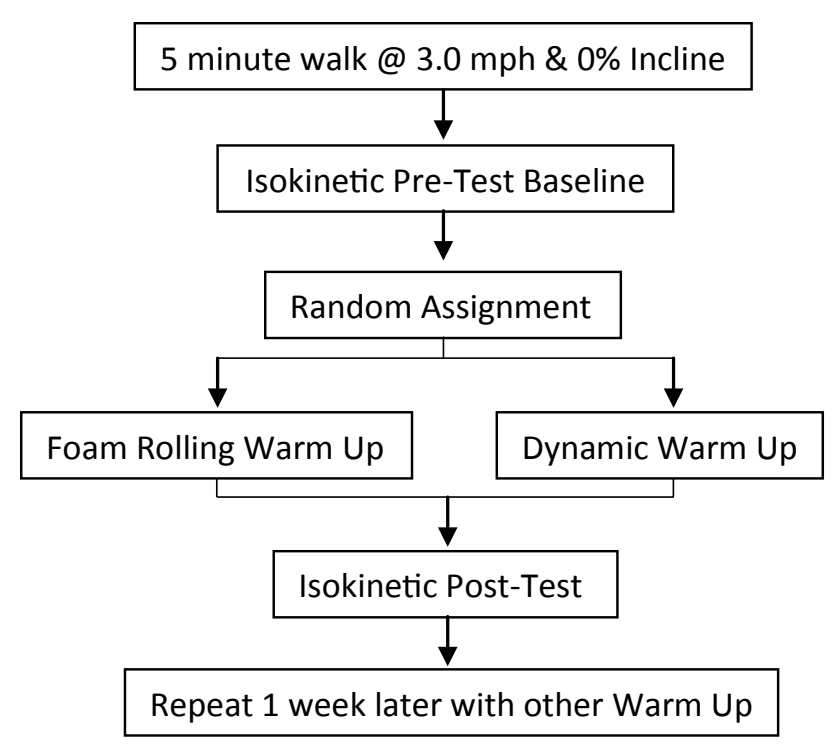

Figure 1: Flow chart of the study.

\section{Procedures}

A 5 minute walk on the treadmill, $3.0 \mathrm{mph}$ and $0 \%$ incline, served as a standard warm-up to prevent injury during the pre-test for both the foam-rolling and active warm-up protocols. Two isokinetic tests on each testing day (i.e. pre and post warm-up) were recorded 
(a)

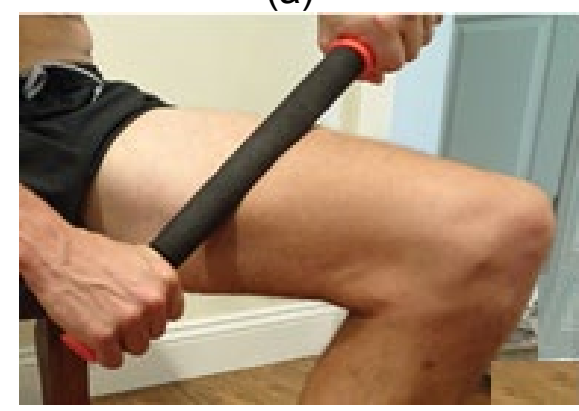

(b)

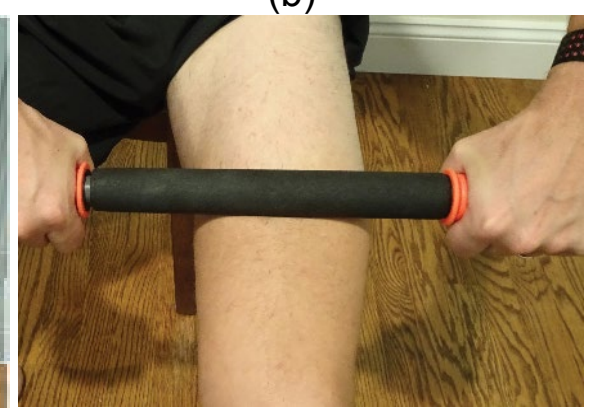

(c)

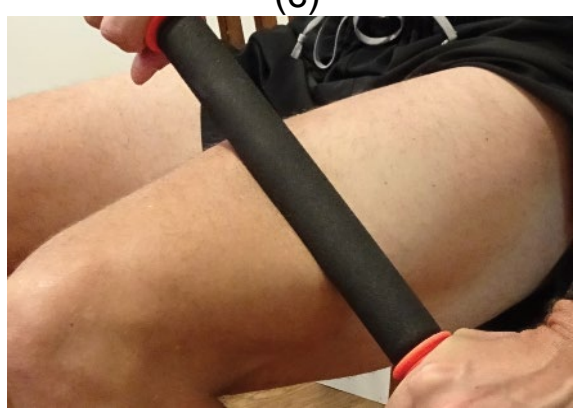

Figure 2: medial (a), anterior (b), and lateral thigh (c).

using a Biodex System 3 (Biodex Medical Systems, Inc., Shirley, NY). Prior to testing, the Biodex chair and tower was set up specifically for each subject according to the machine's protocol guidelines. These settings were recorded to ensure that the chair alignment was replicated for the second testing day. The pre and post-testing required 1 set of 5 repetitions of maximal voluntary contraction of the right quadriceps via knee extension. Approximately 90 degrees of knee extension was performed at 60 degrees per second. Each repetition was counted aloud, and verbal encouragement was provided. The subject's right quadriceps musculature was tested for peak torque, average peak torque, total work and average power. The order of experimental warmup (foam-rolling vs. active warm-up) was randomized for each subject.

\section{Active warm-up protocol}

The active warm-up protocol used in this study was created to focus on the quadriceps and was performed directly after the isokinetic pre-test. It consisted of three active movements: High knees, anterior-posterior leg swings, and lateral leg swings $[9,18,19]$. Three sets of these exercises, in the order listed, were performed with each exercise lasting 30 seconds. There was a $30 \mathrm{sec}-$ onds standing break between each set. The total time of the active warm-up was 4.5 minutes; total rest time during the warm-up was 1.5 minutes. The 4.5 minutes of active exercise was selected to allow for a physiological response that would not cause peripheral fatigue. Past research indicated that a minimum of 3-5 minutes was required to adequately warm-up the body [1].

Subjects were instructed to act as if they were warming up for their sport practice to mimic a similar intensity. The Borg physical exertion scale was used to provide a quantitative measurement of the exertion from each subject during the warm-up protocols. The goal Borg rating was set at $15=$ 'hard'. If they provided a number less than 15, the subject was instructed to increase the intensity of the next set. Similarly, they were told to reduce the effort if the subject provided a Borg rating higher than 15. The Borg rating was taken immediately after completion of each set, and the appropriate feedback of exercise intensity was provided to the subject.
A 2 minute rest period was given to each subject after the completion of the entire active warm-up; during this rest the subject was sitting on the Biodex chair.

\section{Foam rolling warm-up protocol}

The foam rolling warm-up was completed using an original Tiger Tail ${ }^{\circledR}$ (Tiger Tail, Kent, WA) hand held, high-density foam roller. Three zones of the quadriceps were foam rolled by the subject: Medial, anterior, and lateral aspects of the thigh. Each of these zones was foam rolled by the subject for $30 \mathrm{~s}$, followed by a $30 \mathrm{~s}$ rest while sitting before beginning the next set. A breakdown of one set was $30 \mathrm{~s}$ medial thighs, $30 \mathrm{~s}$ anterior thighs, $30 \mathrm{~s}$ lateral thigh, and $30 \mathrm{~s}$ rest (Figure 2). Past research on foam rolling typically adopted a protocol of rolling one muscle group for an average of 1 minute $[9,13,15]$, but as an active warm-up requires 3-5 minutes [1], the total time spent foam rolling was increased to 4.5 minutes with 1.5 minutes of sitting rest, to mirror the time spent performing the dynamic movements. The subjects were instructed to foam roll vigorously with a good steady tempo and a medium amount of pressure. The Borg scale was used to measure exertion of each set, and feedback was provided throughout the warm-up. A two-minute recovery was given to each subject after the completion of the foam rolling warm-up; during this time the subject was sitting on the Biodex chair (Figure 2).

\section{Statistical analysis}

The data was analyzed using SPSS v20.0 (SPSS Inc., Chicago, IL). A total of 8 repeated measures, one-tail t-tests were used to evaluate differences between pre and post-test data (4 dependent variables for both warm-up protocols). It was expected that after each warm-up protocol, the muscles would generate a greater force output. Four two-tail equal variance t-test were used to determine significant differences in changes (post-test minus pre-test) measures between warm-up protocols. The $p$-value was set at 0.01 to account for the 12 total t-tests that were performed.

\section{Results}

Four pre versus post-test measures were evaluated 
Table 1: Mean and standard deviation values for each of the four measures of the Dynamic protocol (DYN).

\begin{tabular}{|l|l|l|}
\hline & Pre-DYN & Post-DYN \\
\hline Average Torque $(\mathrm{N} \cdot \mathrm{m})$ & $107.8 \pm 28.4$ & $121.2 \pm 23.1$ \\
\hline Peak Torque $(\mathrm{N} \cdot \mathrm{m})$ & $130.8 \pm 23.2$ & $137.6 \pm 23.2^{*}$ \\
\hline Total Work $(\mathrm{J})$ & $412.1 \pm 144.3$ & $467.6 \pm 123.8$ \\
\hline Average Power (Watts) & $111.3 \pm 27.7$ & $123.8 \pm 37.9$ \\
\hline
\end{tabular}

*denotes a significant difference $(p<0.01)$ between pre and post measures.

Table 2: Mean and standard deviation values for each of the four measures of the Foam Rolling Protocol (FR).

\begin{tabular}{|l|l|l|}
\hline & Pre-FR & Post-FR \\
\hline Average Torque $(\mathrm{N} \cdot \mathrm{m})$ & $117.2 \pm 26.0$ & $121.0 \pm 27.9$ \\
\hline Peak Torque $(\mathrm{N} \cdot \mathrm{m})$ & $132.0 \pm 22.9$ & $140.7 \pm 22.1^{*}$ \\
\hline Total Work $(\mathrm{J})$ & $464.7 \pm 103.1$ & $474.8 \pm 105.9$ \\
\hline Average Power $($ Watts) & $121.5 \pm 30.6$ & $129.1 \pm 35.1$ \\
\hline
\end{tabular}

"denotes a significant difference $(p<0.01)$ between pre and post measures.

Table 3: Post-test minus pre-test mean and standard deviation values for each of the four measures of the Dynamic (DYN) and Foam Rolling (FR) protocol. 95\% confidence intervals are shown in parenthesis. No significant differences between DYN and FR were found $(p>0.01)$.

\begin{tabular}{|l|l|l|}
\hline \multicolumn{1}{|c|}{ DYN } & FR \\
\hline Average Torque $(\mathrm{N} \cdot \mathrm{m})$ & $13.3 \pm 25.7[-2.3,24.3]$ & $3.8 \pm 27.0[7.7,15.3]$ \\
\hline Peak Torque $(\mathrm{N} \cdot \mathrm{m})$ & $6.8 \pm 23.2[3.1,16.7]$ & $8.6 \pm 22.5[1.0,18.2]$ \\
\hline Total Work $(\mathrm{J})$ & $55.5 \pm 134.1[1.9,112.9]$ & $10.1 \pm 104.5[34.6,54.8]$ \\
\hline Average Power $($ Watts) & $12.5 \pm 32.8[1.5,26.5]$ & $7.6 \pm 32.9[6.5,21.7]$ \\
\hline
\end{tabular}

for both the Active Warm-Up (AWU): Average Torque (AT), Peak Torque (PT), Total Work (TW), and Average Power (AP). Table 1 displays mean and standard deviation results for each measure recorded for the AWU protocol. There were no significant differences for AT (t (20) $=1.1, \mathrm{P}=0.14)$, TW (t $(20)=0.6, \mathrm{P}=0.29)$, or AP (t (20) $=1.7, P=0.10$ ) between pre and post measures. Peak torque, however, showed a significant increase post AWU (t $(20)=4.7, P<0.01)$. Although AT, TW, and AP did not significantly differ, there was a general increase from pre to post results for all four measures (Table 1).

The same four measures, AP, PT, TW, and AT were evaluated for the Foam Rolling (FR) warm-up protocol. The means and standard deviations for each measure are provided in Table 2. Similar to the AWU, following the foam rolling warm-up a significant increase in PT $(t$ $(20)=3.1, P<0.01)$ was found. No significant differences for AP $(t(20)=2.0, P=0.03)$, TW $(t(20)=2.5, P=0.01)$, or AT $(\mathrm{t}(20)=2.4, \mathrm{P}=0.01)$ were found between pre and post-test measures. There was a slight trend of an increase in performance post foam-rolling protocol for these three measures, but not as pronounced as with the AWU protocol.

The change from pre to post values was calculated by subtracting the pre warm-up value from the post warmup value for each protocol separately (Table 3 ). There were no significant differences between the changes of the two groups shown by a two-tail t-test. AT, TW, and $A P$ all revealed no significant differences between $A W U$ and $F R(t(20)=-1.8, P=0.85),(t(20)=-1.7, P=0.1)$, and $(t)(20)=-0.6, P=0.5)$ respectively. The negative t-statistic indicates that average change for the active protocol was higher for the AWU than for FR. PT was the only measure in which FR had the higher average change compared to active, though not significant $(\mathrm{t}(20)=0.7$, $P=0.5)$.

\section{Discussion}

The purpose of this study was to evaluate foam rolling's ability to act as a novel warm-up protocol designed to increase performance. All measures revealed a slight increase from pre to post values across both warm-up protocols, which is indicative of an effective warm-up. This trend, although mostly nonsignificant, provides potential for foam rolling to be considered as a warm-up aid.

\section{Active warm-up}

The Active Warm-Up (AWU) protocol elicited a significant increase in peak torque of the quadriceps musculature. Fletcher and Jones [18] previously evaluated the impact a high knees exercise, along with other dynamic exercises, has on sprint performance. The group of dynamic exercises used in their study significantly increased sprint performance of their participants. Although Fletcher and Jones did not utilize any form of leg swings at the hip, Aguilar, et al. [20] found similar positive findings to the current study with dynamic hip extension stretches. In contrast to the current study, Herda, et al. [19], did not find any significant differences pre to post peak torque of the hamstrings following a dynamic stretch. Their protocol involved three dynamic stretches, similar to the current study, but the stretches were performed in a slower, more controlled manner. The slower movements performed in the Herda, et al. [19] study may not have been sufficiently intense to cause aphysiological benefit. 
The physiological responses of a dynamic stretch during an active warm-up includes, increased body temperature, post-activation potentiation, decreased fascial stiffness, could be the mechanisms that produced the increase in peak torque $[8,20]$. Although these factors were not directly measured, the AWU routine caused a physiological change to stimulate increased performance results. The multitude of theories on the mechanisms behind active warm-up's effectiveness appear to be demonstrated in the current study $[8,18-20]$. The current study also implemented only a three exercise protocol of dynamic stretches. Three exercises were selected, instead of a greater number, to mirror the FR protocol that utilized three bouts of rolling (three zones) per set. The provided a better consistency in time spent warming-up between both protocols.

Overall, all participants showed small improvements in average power and total work. This trend is based on how power and work are derived. Torque, which is a rotational force, is a main component of the formulas for both power and work. Work, the measurement of force over a certain distance, increases as the amount of force increases. Also, power, the amount of force over time, can be directly manipulated by a change in force output. The results of the current study generally follow these mathematical equations. Due to the significant increase in peak torque, both work and power also showed increases post active warm-up. The general trend of all four measures provides continued support for the use of dynamic stretching and active warm-ups for performance enhancement.

The primary limitation of the study was to objectively gauge participant's effort level. During this study, the amount of pressure placed on the quadriceps via the foam roller may vary between participants as well as between testing sessions within a participant. The dynamic warm-up may also have tempo and range of motion variants among the participants. A Borg scale and verbal encouragement were utilized to minimize the amount of participant variability; all participants were also in structed on proper technique for each protocol using the same vernacular.

\section{Foam rolling}

With the limited research on foam rolling during a warm-up, little can be compared to the current findings. The justification behind using a hand-held foam roller to warm-up the lower body is directly related to energy usage. If a participant can effectively warm-up the lower body using the upper body musculature, then in theory no energy substrates would be removed from the target tissues. This preservation of energy could potentially prolong anaerobic efforts due to more substrates being available.

Similar to the active warm-up, all measures appeared to have a slight increase from pre to post measurements using the foam rolling warm-up. The statistical outcome indicated only a significant difference between the four pre and post measures for foam rolling. The statistically significant increase in peak torque suggests that foam rolling can be a beneficial form of pre-performance preparation.

Healey, et al. [15] found similar results to the current study using body weight foaming rolling compared to a planking exercise. The study utilized the body weight plank exercise as a control because it closely mimicked the style of foam rolling used in that study. Foam rolling on the floor using one's body weight requires more effort than using a hand held foam roller as the individual must support their body weight as they roll back and forth. The planking warm-up, however, would not have caused any effects along the specific muscle groups themselves. Although the 5 athletic tests used in the Healey, et al. study are not directly related to the current study's measures, a comparison can be made in that there was no significant differences in general performance between the two interventions. All participants in that study also showed a general increase from pre to post performance regardless of the warmup methods.

Similar to Healey, et al. [15], Peacock, et al. [9] used athletic tests, with the addition of a flexibility test, to determine the effects of foam rolling. The study combined an active warm-up with a foam rolling protocol of five muscles for 30 seconds one time through. The results of the study showed a statistically significant increase in vertical jump and long jump performance following the combination of active and FR when compared to active alone [9]. In contrast, Macdonald, et al. [13] found no increase in voluntary muscle activation following 2 sets of 1 minute bouts of foam rolling of the quadriceps. All bouts of foam rolling in these three studies were similar in their total duration and intensity, but Macdonald, et al. [13] utilized slightly longer bouts of foam rolling and less repetitions.

Foam rolling could be considered an alternate form of static stretching due to its innate ability to increase range of motion [13]. However, in respect to performance, static stretching and foam rolling appear to have contradicting effects. Many studies have shown that static stretching causes a decrease in power $[6,8,21,22]$, but so far, with the addition of the current study, foam rolling does not appear to have any negative effects $[9,13,15]$. This could potentially be related to a lack of true increased sarcomere length with foam rolling, which is the current theory on decreased power following static stretching. Malin, et al. [23] found confounding results when measuring peak power output following static stretching in combination with FR. Static stretching, as expected, caused a drop in power among the female group, but both static stretching and foam rolling caused an increase in power among the males. The tissue elasticity seen in the Macdonald, et al. [13] study could be 
due to increase blood flow and/or increase muscle fascia elasticity. This theory would support the current results in that the foam rolling exercise is not causing neuromuscular inhibition because there are no sufficient length changes taking place to activate the neuromuscular inhibitors.

Foam rolling has also been used as a substitute to massage and myofascial release [15], but it does not appear that massage, myofascial release, and foam rolling have similar effects on performance based on the findings in the current study. Massage has been traditionally used to induce muscle relaxation, reduce tension, relieve soreness, promote healing, and theoretically improves performance; however it is more so to aid in recovery [12]. In the area of sport pre-performance massage, little research reveals positive outcomes. Goodwin, et al. [24] examined 30 meter sprint times following a 15 minute lower-body massage. No statistically significant changes were found between the experiment and control groups. However, Wiktorsonn-Moller, et al. [25] found that a 15 minute massage yielded decreased performance on an isokinetic dynamometer. Myofascial release (MFR), as with massage, shows conflicting results; Kuruma, et al. [26] demonstrated significant decreases in reaction time following MFR on the hamstrings and quadriceps musculature. It was unclear of the impact foam rolling would have on performance, but as the technique is a combination of modalities, it was theorized it would provide the physiological benefits without the performance decrements.

The mechanisms behind foam rolling are still unclear, but its use as a therapeutic modality and now performance modality has become more common. Over all, there were no significant decreases in performance following foam rolling. However, the significant increase in peak torque, and over all general pre-post increase trends, shows promise for foam rolling as an aid for performance enhancement. Increased tissue elasticity, increased blood flow, increased muscle and skin temperature, and increased range of motion are potential benefits from a proper warm-up to facilitate performance. All of these physiological responses have been shown to result from massage and MFR, but the past research does not support their use for performance enhancement $[12,13]$. Therefore, foam rolling should be categorized and referred to on its own without being grouped together with MFR or SMR.

\section{Between groups}

Peak torque for both the AWU and FR groups statistically increased; the changes were 6.8 and $8.6 \mathrm{~N} \cdot \mathrm{m}$ respectively. This increase in peak torque, as described above may have caused the increase in power and work as well, due to their mathematical relationship. The AWU and FR groups' revealed similar results in all aspects, which assist the theory that FR could be a beneficial method for warming-up.

The active movements and dynamic stretches were selected based on their similarity to the foam rolling techniques, by targeting the quadriceps. High knees, anterior-posterior leg swings, and lateral leg swings directly affect each aspect of the quadriceps musculature. The current study did not utilize active warm-up techniques such as jogging, sprinting, or plyometric exercises as they are more cardiovascular in nature and a direct comparison to the FR warm-up would be more difficult. The goal of the AWU was to elicit physiological changes including increased blood flow and range of motion, without causing fatigue of the tested muscles. When asked, the participants used words like: Sweaty, warm, winded, and pumped, to describe how the AWU made them feel. When asked to describe how the FR exercises made them feel, they described warmth of the skin and tissues of the thigh, fatigue of the upper body, and increased vascular activity. On the lighter-skinned participants, rubor of the skin over the anterior thigh was noticeable during and immediately following FR. A total body rubor was noted on a few of the participants following the AWU. These outcomes suggest similarities in physiological responses between the two warm-ups.

\section{Conclusion}

Overall, the current study reveals that FR is comparable to dynamic stretching during an active warm-up which suggests FR has more potential uses than simply as a rehabilitation modality. Research seems to indicate that FR yields positive outcomes when used before exercise. Also, FR could be categorized as an active warm-up due to the work performed by the upper body (or whole body with the floor FR). Therefore, foam rolling could act as an alternative to dynamic stretching during an active warm-up. The current study suggests that foam rolling is comparable to dynamic stretches and perhaps the two in combination would provide the greatest warmup outcomes, though this has yet to be confirmed.

\section{Acknowlegements}

The authors would like thank the members of the soccer, football and basketball teams who volunteered to participate in the study. Thanks also go to the Human Motion Research Lab and the Exercise Physiology Lab at William Paterson University for their equipment and resources.

\section{References}

1. Bishop D (2003) Warm up I: Potential mechanisms and the effects of passive warm up on exercise performance. Sports Med 33: 439-454.

2. Fradkin AJ, Zazryn TR, Smoliga JM (2010) Effects of warming-up on physical performance: A systematic review with meta-analysis. J Strength Cond Res 24: 140-148.

3. Shellock FG, Prentice WE (1985) Warming-up and stretching for improved physical performance and prevention of sports-related injuries. Sports Med 2: 267-278.

4. Bishop D (2003) Warm up II: Performance changes following active warm up and how to structure the warm up. Sports Med 33: 483-498.

5. Church JB, Wiggins MS, Moode FM, Crist R (2001) Effect 
of warm-up and flexibility treatments on vertical jump performance. J Strength Cond Res 15: 332-336.

6. Behm DG, Button DC, Butt JC (2001) Factors affecting force loss with prolonged stretching. Can J Appl Physiol 26: 261-272.

7. Cramer JT, Housh TJ, Johnson GO, Miller JM, Coburn JW, et al. (2004) Acute effects of static stretching on peak torque in women. J Strength Cond Res 18: 236-241.

8. McMillian DJ, Moore JH, Hatler BS, Taylor DC (2006) Dynamic vs. static-stretching warm up: The effect on power and agility performance. J Strength Cond Res 20: 492-499.

9. Peacock CA, Krein DD, Silver TA, Sanders GJ, VON Carlowitz KA (2014) An Acute Bout of Self-Myofascial Release in the Form of Foam Rolling Improves Performance Testing. Int J Exerc Sci 7: 202-211.

10. Prentice WE (2010) Principles of Athletic Training: A Competency-Based Approach. (14 ${ }^{\text {th }}$ edn), McGraw-Hill Education, Columbus, USA.

11. Sefton J (2004) Myofascial Release for Athletic Trainers, Part 1. Athl Ther Today 9: 48-49.

12. Weerapong P, Hume PA, Kolt GS (2005) The mechanisms of massage and effects on performance, muscle recovery and injury prevention. Sports Med 35: 235-256.

13. MacDonald GZ, Penney MD, Mullaley ME, Cuconato AL, Drake CD, et al. (2013) An acute bout of self-myofascial release increases range of motion without a subsequent decrease in muscle activation or force. J Strength Cond Res 27: 812-821.

14. Hough PA, Ross EZ, Howatson G (2009) Effects of dynamic and static stretching on vertical jump performance and electromyographic activity. J Strength Cond Res 23: 507-512.

15. Healey KC, Hatfield DL, Blanpied P, Dorfman LR, Riebe D (2014) The effects of myofascial release with foam rolling on performance. J Strength Cond Res 28: 61-68.

16. Curran PF, Fiore RD, Crisco JJ (2008) A comparison of the pressure exerted on soft tissue by 2 myofascial rollers. J Sport Rehabil 17: 432-442.
17. Okamoto T, Masuhara M, Ikuta K (2014) Acute effects of self-myofascial release using a foam roller on arterial function. J Strength Cond Res 28: 69-73.

18. Fletcher IM, Jones B (2004) The effect of different warm-up stretch protocols on 20 meter sprint performance in trained rugby union players. J Strength Cond Res 18: 885-888.

19. Herda TJ, Cramer JT, Ryan ED, McHugh MP, Stout JR (2008) Acute effects of static versus dynamic stretching on isometric peak torque, electromyography, and mechanomyography of the biceps femoris muscle. J Strength Cond Res 22: 809-817.

20. Aguilar AJ, DiStefano LJ, Brown CN, Herman DC, Guskiewicz KM, et al. (2012) A dynamic warm-up model increases quadriceps strength and hamstring flexibility. J Strength Cond Res 26: 1130-1141.

21. Behm DG, Bambury A, Cahill F, Power K (2004) Effect of acute static stretching on force, balance, reaction time, and movement time. Med Sci Sports Exerc 36: 1397-1402.

22. Bradley PS, Olsen PD, Portas MD (2007) The effect of static, ballistic, and proprioceptive neuromuscular facilitation stretching on vertical jump performance. J Strength Cond Res 21: 223-226.

23. Malin B, Jordan $M$, Cook R, Hagenbucher J, Draeger A, et al. (2013) Effects of Self Myofascial Release \& Static Stretching on Anaerobic Power Output. J Fit Res 2: 2.

24. Goodwin JE, Glaister M, Howatson G, Lockey RA, Mclnnes $\mathrm{G}$ (2007) Effect of pre-performance lower-limb massage on thirty-meter sprint running. J Strength Cond Res 21: 10281031.

25. Wiktorsson-Möller M, Oberg B, Ekstrand J, Gillquist J (1983) Effects of warming up, massage, and stretching on range of motion and muscle strength in the lower extremity. Am J Sports Med 11: 249-252.

26. Kuruma $H$, Takei $H$, Nitta $O$, Furukawa $Y$, Shida $N$, et al. (2013) Effects of myofascial release and stretching technique on range of motion and reaction time. J Phys Ther Sci 25: 169-171. 